group of confluent vesicles, as would be conferred by making four separate scarifications over smaller areas. Explanations are a source of comfort to those who can accept them and of vexation to those who cannot. In any case they are of secondary importance and if the public can be induced to recognise that four large scars are associated with a higher state of protection than one small scar and to condemn the practice of vaccination by less than four insertions they need not be burdened with the explanation of these mysteries.

\section{A NOTE ON COLOTOMY AND COLECTOMY.'}

\section{BY F. T. PAUL, F.R.C.S. ENG.,}

SURGEON TO THE LIVERPOOL ROYAL INFIRMARY; PROFESSOR OF MEDICAL JURISPRUDENCE AND LEOTURER ON DENTAL ANATOMY AT UNIVERSTTY COLLEGE, LIVERPOOL.

SoMe ten years ago the value of colotomy in cases of recta cancer was discussed at this society. At that time several of the most experienced members of the Liverpool Medical Institution were only prepared to adrocate it as a means of giving relief when the most urgent and distressing symptoms were present. I remember that it was classed with gastrostomy and gastro-enterostomy for malignant stricture as dangerous to life and as producing other ills almost, if not altogether, as unpleasant as those which it was designed to relieve. We may truly say now that we have changed all this, not only for colotomy, but for gastrostomy and gastroenterostomy as well, but especially as regards colotomy. And it is felt now that it is right to recommend the latter in any case of rectal cancer unsuitable for excision in which distressing symptoms are present or are indicated ; indeed, enthusiasts (and I may include myself) for high operations in extensive rectal cancer seem to be inclining again to substitute a permanent iliac colotomy for these very serious and wide operations, or to do it as a preliminary, and so to lessen the risk of the subsequent proceeding.

Modern colotomy has unquestionably gained in favour, and this gain is due to two causes: (1) lower mortality and (2) improved artificial anus. The latter has, I consider, been the more important factor, for when a patient is labouring under a mortal disease it is more a question of comfort and the capacity to continue his daily arocation than the simple prolongation of a more or less miserable existence. In the discussion to which I have referred it seemed to be strongly felt that the discomforts of the artificial anus were frequently as great as those of the malignant ulceration. The want of control over the evacuations, the dependence upon assistance after each movement of the bowels, and the rather frequent occurrence of prolapse certainly rendered the life of a colotomy patient a far from happy existence. Now usually very fair control is established in most cases, and prolapse hardly ever occurs. whilst with an anterior opening the patient can attend to himself-a condition of independence which is greatly appreciated by the good, hopeful, deserving class of patients. I have no sympathy with those who give in. It is the duty of all of us, not only to live, but to work as long as we are able, and it is as we all know quite possible for any colotomy patient not otherwise disabled to do his daily work, whether manual or sedentary, when the artificial anus is a good one.

Amongst the various suggested methods for performing iliac colotomy it must be conceded that, at any rate in England, Cripps's method is that which has met with most general favour ; indeed, Mr. Herbert Allingham, who advocated a rival method, has of late accepted the former except in cases in which the sigmoid mesentery is long and the patient may be expected to survive a considerable time. In my judgment Allingharn's present position is a wise one. The variability of the anatomical conditions of the sigmoid flexure is such as to render it impossible that the same method of dealing with it can be the best for every case, and it certainly seems the fact that when the flexure is voluminous and its mesentery long Cripps's method, however carefully accomplished, is very likely to be followed by a moist patulous anus, over which it is diflicult to create any control and through which prolapse of bowel is very probable. Allingham's procedure is undoubtedly rather more severe, but it results in an excellent orifice and the increased

paper read at the Liverpool Medical Institution on Feb. 12th, comfort when the operation is properly indicated is well worth the increased risk. 'The results desired from a colotomy are (1) relief, (2) safety, and (3) comfort.

First, as to relief. In cases of obstruction immediate relief is urgent. This perhaps applies to 20 per cent. of the cases; less when the disease is in the rectum; but more when it is in the colon. When the operation is undertaken with the necessity of giving immediate relief I urge the glass-tube method, and I may do this with more confidence now than in 1891 when I first introduced it, since not only has my personal experience of the method been fairly considerable since that time, but I gather that it is now generally practised in these circumstances. Cripps's and Allingham's operations do not provide for the immediate opening of the bowel; but I have practised both operations many times, and in all sorts of circumstances, in conjunction with the glass tube, and have no reason to think that these operations are rendered appreciably more dangerous thereby. If this experience is correct and it is true-as I believe it to be - that opening the bowel at the time of the primary operation by the glass-tube method does not appreciably increase the risk to life, then it is certainly a desirable addition in most cases, for not only is it convenient that the bowel should be open and free for the passage of flatus or fæces, but the wound in the abdominal wall may be smaller, a point of some importance, and the operation is completed at one sitting. There is no occasion for the subsequent incision and later paring of the mucous membrane required in Cripps's operation, and there are no subsequent clamping and removal of bowel as in Allingham's. I therefore distinctly recommend that in performing either of these operations the glass tube should be used and the bowel opened at once, whether obstruction is present or not.

Next as regards safety. When the patient is in an exhausted condition Cripps's operation with or without the tube should be selected, as the removal of bowel, especially if the mesentery be thick, increases the risk. Should obstruction be present in introducing the tube every precaution must be taken to avoid leakage. In all cases both the bowel and the tube should be attached to the abdominal wall, the bowel by at least two green-gut sutures connecting it with the deep muscular aponeurosis, and the tube by passing its silk ligatures through the skin and knotting them. Then if the tube is accidentally dragged on it is well and safely anchored and when it comes away, if the adhesions between the bowel and the parietal peritoneum are weak, they are backed up by the green-gut satures.

As regards comfort the essentials are : (1) a high operation, a small wound, and opening the highest portion of colon available, as recommended by Cripps; (2) the formation of a good spur; (3) embodying the principle of Allingham's operation whenever the sigmoid flexure is voluminous and its mesentery long and the patient sufficiently robust to make this wise; and (4) the use of a good plug and the cultivation of a regular habit of bowels. After various trials with trusses and belts $I$ have come to use an aluminium mushroom-shaped plug. The plug or stalk is slightiy bulbous and long enough to pass through the whole depth of the abdominal wall. The head or flange is oval-shaped and wide; it is slightly concavo-convex to fit the abdominal surface. A single layer of soft lint with a hole in the middle for the stalk is placed under the flange and a good pad of cotton-wool above it, the whole being kept in place by a narrow abdominal belt, such as I use after appendicectomy. This is the cleanest and by far the most comfortable and effective apparatus $I$ have yet tried.

In $1898 \mathrm{I}$ designed an operation to give better control over the artificial anus. It involved using Madelung's method of closing and returning the rectal end of the bowel into the abdominal cavity, whilst the upper end was brought out at a distance, after having passed between the muscles-in fact, after the principle of Hahn's or Frank's gastrostomy. The operation was effective but dangerous. It was unnecessarily severe and in passing the bowel between the muscles septic infection was liable to occur. For these reasons I soon abandoned it and I think now that sufficiently good control can be obtained by ordinäry methods.

The details of a colotomy by the glass-tube method are as follows. 1. The incision is made in accordance with accepted principles, but may be smaller, that is from one and a half to two inches, according to the condition of the abdominal wall and the bowel. 2. If the mesentery be very short the bowel is firmly drawn out and a running suture of medium thick silk is inserted round an oval area involving 
as much of the circumference of the bowel as possible, so as to insure its posterior wall being well brought up to the surface to form a good spur. The inclosed area is then gripped with two catch forceps, incised, the tube plugged with wool inserted, and the ligature tied. $\mathbf{A}$ second silk ligature is then simply tied over the first to render it more secure and is knotted on the opposite side of the tube. Two or more green-gut sutures are next passed between the outer coats of the bowel and the deep tissues of the wound, and finally the ends of the silk ligatures are passed through the skin and tied so as to secure the tube in case of roughness or accident. Usually the wound is too small to need any further sutures. The parts are well powdered with iodoform and dressed with gauze and wool as usual, and a binder split to transmit the tube is firmly applied. If the bowel is empty a light plug of wool is kept in the tube. If loaded with formed motion a sponge bag containing some iodoform wool or wool sprinkled with eucalyptus oil is attached. If with loose motion a short piece of Down Brothers' thin wide indiarubber tubing is attached to the glass-tube and allowed to fall into a basin at the patient's side. 3. If the mesentery be long and the patient in good condition the loose loop of sigmoid flexure should be drawn out, the mesentery ligatured separately, a large tube fastened into the top end and a small one into the bottom end, and then all the redundant bowel cut away. It is better not to ligature the mesentery together with the bowel to the tubes, as unless the grip is very tight some of the vessels are liable to retract and smart hæmorrhage may result. It will be found much safer to tie the mesentery in two or three sections by itself. When the sigmoid mesentery is of medium length either Cripps's or Allingham's method may be used; but in all cases when doing Cripps's operation care must be taken to insert the tube into the highest portion of bowel which can be brought out of the wonnd and to include the whole, or almost the whole, circumference of the bowel. 4. Usually on the fifth day the tube is ready to come away. When this is the case, indicated by leakage beside the tube, the silk sutures are cut and it is withdrawn. 5. Subsequently the patient is kept in bed, the motions being passed into the dressings until the wound is healed, when the plug and belt are worn.

Colotomy in other regions.-For some time I maintained it was our duty to perform colotomy as low down in the bowel as we could consistently with being well clear of the disease. Now I am inclined to think that there are only two good colotomies-sigmoid and transverse. In the few cases in which I have had to do the latter it has answered very fairly well-not so well as the sigmoid but decidedly better, I consider, than the lumbar operation. Right iliac colotomy I do not like at all; the motions are liquid and quite uncontrollable. I never do it except in cases of obstruction and then only in the hope and expectation that subsequent enterectomy or short-circuiting wiil be possible. When the cæcum is opened only a small tube should be used, one of from half an inch to five-eighths of an inch in diameter being sufficient.

It has been said that subsequent trouble will result if either the sigmoid flexure or cæcum are opened and attached in the middle line. I have had to do this occasionally and in practice no inconvenience has resulted. This position would never be chosen, but at any time there may be urgent reasons why it should be adopted.

The operation of colotomy is so closely associated with that of colectomy that I can hardly treat of one without reference to the other. Often when colotomy only is intended before exploration colectomy is ultimately undertaken, or the reverse may be the case; whilst, as $I$ have stated, it is no unusual thing for me to cut away several inches of colon with the simple object of improving the character of an artificial anus. When, however, the original intention is colectomy there are a few additional points which need careful consideration. The chief of these are the exact location of the disease and the restoration of the continuity of the bowel.

It is a great misfortune that malignant disease of the colon is so very undemonstrative in early stages. Recently in one week I was asked to see three cases all of which were too late for operation-that is, in all the tumour was hopelessly adherent to neighbouring important structures. This form of cancer is one of the least malignant with which I am acquainted, and could one obtain the cases early enough, as happens more frequently in the rectum, the results would be excellent. In its first stage, however, it apparently sets up no recognisable symptoms and I have no doubt it often exists for several months or a year or two, according to the type of the growth, before any evidence whatever of its presence is afforded. The earliest symptoms are frequently those of intestinal indigestion, brought about by some difficulty in the contents of the bowel passing the diseased part; they are flatulence, slight colicky pains, cases there are no obstructive symptoms at first, but mucus and perhaps blood appear in the motions. At a later stage some obstruction is usually present, characterised by increasing constipation with attacks of colicky pain. Loss of flesh occurs and ultimately a tumour is discovered in some cases, whilst in others the surgeon is called in because com. plete obstruction has supervened. The clinical details are influenced by the character of the growth. From 35 to 45 or 50 years of age the disease is generally of the fungating type and produces more of a tumour than an ulcer. In these cases there is often no obstruction but plenty of mucus and blood in the motions. The abdomen is not distended and if carefully palpated the tumour might usually be discovered much earlier than it is. Still, one must bear in mind that the patient suffers little and frequently makes no com. plaint until it is too late. Indeed, it is sometimes the discovery of the tumour by the patient himself that leads him to consult his medical adviser. From 50 to 70 or 80 years of age the growth is usually of the chronic ulcerating type, producing the ring stricture. In these cases the symptoms are almost entirely due to obstruction, which in the end generally becomes complete owing to the narrow lumen at the strictured part (often only transmitting a No. 10 catheter) becoming blocked by some undigested substance in the fæces.

When distension is absent a tumour is often present and in this way the disease is usually located in the younger class of patients ; but after obstruction has once set in it is rare to be able to detect a lump and one must be guided in other ways in locating it. Probabilities are of no help. The surgeon must never cut down over a particular region because that is statistically the most probable site of the disease. In the absence of stronger evidence "exploration in the middle line should be the practice. The best aids to diagnosis are the capacity of the bowel below the stricture, the condition of the creum and colon as made out by percussion and palpation, and the evidence afforded by pain and peristalsis. The patient is often quite conscious of the site of the stricture, having many a time felt a colicky wave of peristalsis pass away at a particular spot with a gurgling sound as gas and liquid were forced through the stricture. And when the bowel is much hypertrophied one may see the peristaltic wave gather and run on until it comes to a stop at the stricture. The enema, too, is a great help when the disease is low down. As the result of experiment on the dead subject I believe the bowel will hold something like two pints with an obstruction in the sigmoid flexure, but in actual practice one finds that from half a pint to a pint is all that is tolerated, usually the former, and that it is returned at once without motion. In these circumstances the tumour is in the most favourable place for operation. When it is higher up large quantities of injection may be introduced and retained. The operator has then to be guided chiefly by palpation and percussion of the regions of the large bowel and if nothing definite can be made out a middle-line exploration should be undertaken.

When the tumour is located it must be freely exposed, and if suitable for removal the mesentery in connexion with the part involved is carefully divided so that the diseased coil of bowel may be brought out of the abdomen. The subsequent steps of the operation depend upon the mode of union to be attempted, and in regard to this I continue to differ strongly from those who consider primary end-to-end union by suture or button the proper course to adopt as a routine practice. That it is the neatest and most satisfactory to the surgeon when the patient lives I do not doubt; but it is certainly more dangerous without ultimately being at all more efficacious than when the ends of the bowel are brought out and the continuity of the canal is subsequently restored. When this is done it is a rare thing to lose a patient who is not at the time of the operation seriously depressed as the effect of several days' obstruction of the bowels. In this connexion I may as a surgeon warn my medical friends that a patient after ten days obstruction in the lower bowel is in much worse case 
than his pulse and appearance seem to indicate. The surgeon is asked to perform colotomy in these circum. stances with the assurance that the patient is in excellent condition. It is quite true that he is fit to bear the operation; but when he dies a few days later death should be ascribed to the absorption of toxins, which is the cause of it, and not to the operation which has had nothing to do with the unfortunate result.

In 1892 I commenced operating by bringing out the ends of the bowel and after ten years' experience am strongly confirmed in my opinion that it is the right thing to do. I know nothing against it except that the patient has the dis. comforts of an artificial anus for a few weeks and the course of the case is prolonged by about a month-small evils com. pared with the gravity of the disease and the risks of end-toend union. It has been suggested that stricture might result from incomplete removal of the spur, but such has not proved to happen in any of my cases. After dividing the mesentery the subsequent steps of the operation are of the simplest. The tumour being well drawn out of the abdomen, the ascending and descending portions of the colon are made to lie side by side, like the barrels of a double-barrelled gun, in which relationship they are lightly sutured together for quite three inches inside the abdomen. Outside the abdomen a colotomy tube is ligatured into the upper end and a smaller tube into the lower end, when the diseased part is cut away, of course quite bloodlessly. A few fine sutures may be used to attach the bowel to the parietal peritoneum at the bottcm of the wound, and if the incision has been long its ends ar brought together with fishing-gut sutures.

In many cases the operation is very littile more severe than colotomy, Five or six days later the tubes separate, and at the end of three weeks, as a rule, the carefully prepared spur may be clamped. I once had a warning that this should not be done too hastily. After complete obstruction the bowel is liable to be in a very septic and irritable condition, and so long as the spur feels thick and hard to the finger I believe it to be unwise to apply the clamp. As a. rule it is supple throughout, and in these circumstances may be clamped at any time. One clamping is very likely to be insufficient, and if there is a considerable ridge stil] apparent at the bottom the clamp should be applied again. It need not be kept on each time until it comes away, as its work is thorougbly 6 ffected in 48 hours, when it is probably safer removed than left in situ. As soon as the spur is com pletely destroyed the artificial anus is closed. The entire operation has been completed in 24 days; but usually from six to eight weeks must be allowed for it.

In cases of malignant disease of the colon in which the mesenteric glands are involved I used to give a most depressing prognosis, but have now met with a few in which such a prognosis was not justified. About four years ago I removed a cancerous tumour of the sigmoid flexure, together with several enlarged glands in the attached portion of the mesentery. The glands were as large as Barcelona nuts, and I thought the case quite hopeless; nevertheless. she is now, four years after the operation, in excellent health and still continuing her vocation as a domestic nurse.

Two years ago I removed the cæcum and lower part of the ileum from a man, aged 38 years, for a soft malignant growth. The lump was of the size of a fist and severa glands were enlarged, one measuring one and a half inches in diameter. Here, again, I gave a bad prognosis. He re covered well from the operation and a pear later was in such excellent health that his medical friend. Dr. F. Heatherley. refused to countenance any longer a pesimistic outlonk. He came to me and said, in effect, "Look here, you must do something for this fellow. He must not be condemned to die in this sort of way. How about the $x$ rays?" Well, I do not think we have at present any information which warrants us to suppose that the $x$ ravs would influence the course of such a case, so I suggected that if the patient was willing to lie up for a month 1 would explore the abdomen, ascertain what the present condition was, and do what seemed to be best in his interests. This was decided on and one day, after quite half an hour's exploration, I succeeded in discovering and extracting one small gland of the size of a split pea which on microscopical examination showed no malignant change. Another year has passed and the patient is still as well as ever-so well, in fact, that he at once agreed to come here with Dr. Heatherley to show us that we know almost as little about the prognosis as about the treatment of cancer.

Liverpool.

\section{CAN THE RESPIRABLE PRODUCTS OF HUMAN WASTE HINDER DEVELOP. MENT AND LOWER VITALITY?}

\author{
BY JOHN HARTLEY, F.R.C.S. ENG.,
}

HOMORARY SURGEON TO THE DARLINGTON HOSPITAI; LATE SEMIOH DFMONSTBATOR OF ANATOMY, MIDDLESEX HOSPITAL MEDICAL SCHOOL.

THE following premisses will scarcely be denied. 1. That the infinite precautions for the due excretion of waste products observable in the economy constitute primâ-facio evidence of the poisonous nature of such products and that any serious or prolonged interference with the functions of any one of the great channels of exit-lung, kidney, bowel, or skin-is invariably followed by death. 2. That the blood current forms the chief medium of dissemination for systemic poisons. 3. That poisoning by the lungs may be at least as rapid and effectual as that by the alimentary canal, as witness the action of the so-called general anæsthetics, of sulphuretted hydrogen, carbon monoxide, and the like. 4. That the common use of the term "insanitary" as applied to ill-drained, ill-ventilated, and crowded dwellings is evidence of a consensus cf opinion that the air of such dwellings has a definite influence in lowering vitality. Probably none of the foregoing statements will be seriously questioned, but when one approaches the crux of the matter ard asks why is an insanitary dwelling insanitary, one receives answers such as the following: "Absence of sunlight," "absence of ozone," "absence of oxygen," "presence of organisms," "overcrowding."

If one ventures to suggest that the volatile products of animal waste may constitute deadly toxins and that such may find a very direct road to the tissues through the lung, one is met by incredulity. I find no suggestion in medical literature that such a process may be common. Volumes, and most useful ones, have been written on the subject of poisoning through the alimentary canal, as well as larger, and perhaps less useful, volumes on diet and drink. Even the theory of autointoxication (apparently a kind of unreasonable tissue-cussedness) has been dragged into account for the phenomena of lowered vitality. A girl has pale cheeks, damp cold hands, feeble pulse, and poor appetite, and she keeps them in spite of our drugs. No organic disease is to be found. Is one to be content with a diagnosis of constitutional debility or even autointoxication? No doubt the former is true so far as it goes. The predisposing cause must be undoubtedly within the girl, but the determining cause, the active poison, as I believe, starts outside the girl and, I think, usually enters by the lung. Here one, of course, is met by the argument, "But all the rest of the household may keep well under precisely similar conditions." To this there is the obvious answer: a single glass of whisky will make some persons drunk; a dose of poison harmless to the many may be harmful to the few. Medical opinion seems to hover on the edge of this question in a curiously uncertain manner. Its discussion is rarely or never attempted. Yet if there is anything in the view that the volatile products of animal waste constitute a poison readily absorbable by the lung, one can scarcely conceive of any more important issue, and it is solely in the hope of provoking some discussion of the subject that $I$ venture to air my own crude ideas.

If we trace the path of a soluble body in its passage from the alimentary canal to the tissues and compare it with that of a volatile toxin starting from the alveolus of the lung, we see that even at the start the former may be handice pped by the possible action of the digestive ferments, then, according to its nature, it must run the gaun let either of the epithelial cell or of the capillary of the liver guarded by the hepatic cell, and lastly it must face the ordeal of the capillary of the lung before reaching the tissues. In the case of the volatile toxin approaching by the lung the only barriers between it and the blood are the thin cellular walls of the air cell and capillary specially designed for the transmission of gases. The poison enters just at the moment that the blood is undergoing its final purification previously to being distributed to the tissues. From the capillary of the lung to the capillary of the tissue the road is clear, all is main line and "la Grande Vitesse." There can be no doubt that the lung provides the royal route 